\title{
APLICABILIDAD DEL RIEGO A PULSOS EN TABLARES
}

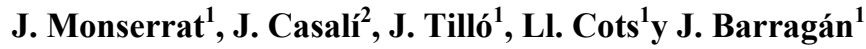 \\ RESUMEN: Se presentan ocho ensayos comparativos entre riego a pulsos y riego conti- \\ nuo en tablares, para variadas condiciones de parcelas y variables de riego. Se ha anali- \\ zado el tiempo de aplicación para llegar al final de la parcela, la lámina infiltrada media, \\ el coeficiente de uniformidad, y el perfil de agua infiltrada. Los resultados muestran que \\ en la mayoría de los ensayos el riego continuo ha mejorado el riego a pulsos. Las condi- \\ ciones en que el riego a pulsos ha resultado mejor son: Caudales unitarios bajos (1.5 l/s/ \\ $m)$, aspereza o rugosidad hidráulica baja $(n=0.02)$ y suelo recién labrado. El factor \\ controlador de la efectividad de los pulsos en riego por tablares parece ser el volumen \\ superficial durante el avance; cuando este es grande, la efectividad disminuye.
}

\section{INTRODUCCIÓN}

La mejora del uso del agua en los regadíos es un importante objetivo en la planificación hidrológica. La técnica del riego a pulsos está siendo crecientemente utilizada en diversos países dadas las mejoras que aporta, según se ha comprobado en diferentes estudios, enfocados fundamentalmente al riego por surcos.

Un riego a pulsos consiste en la aplicación intermitente de agua, originándose como consecuencia periodos alternados de humectación y de secado sobre la superficie regada (USDA-SCS, 1986). A cada una de esas aplicaciones que se realiza intermitentemente se le denomina pulso. Los periodos de humectación o secado pueden ser de duración constante o variable, intentándose siempre que se produzca un receso completo entre una aplicación y la siguiente. En la mayoría de los casos, el agua es aplicada alternativamente entre dos parcelas contiguas hasta que el riego se completa en ambas. La aportación de agua puede realizarse mediante una válvula especial generadora de pulsos con controlador automático.

El efecto positivo del riego a pulsos se explica por la reducción en la velocidad de infiltración que se consigue con la alternancia sobre un suelo de periodos de humectación y de secado. Esta reducción en la velocidad de infiltración se traduce en una mayor cantidad de agua dispuesta sobre la superficie del suelo, lo que hace que el avance sea más rápido. $\mathrm{Al}$ ser el avance más rápido y ser menor la diferencia entre los tiempos de contacto agua-suelo que se dan entre los extremos aguas arriba y aguas abajo de la parcela, aumenta la uniformidad. Además, como disminuye la infiltración, las pérdidas por percolación pueden controlarse mejor, lo que podría traducirse en un aumento de la eficiencia de la aplicación. Múltiples ensayos han puesto de manifiesto la eficacia del riego a pulsos en surcos en variadas circunstancias. Puede encontrarse una buena revisión en Walker y Skogerboe (1987).

Sin embargo, el riego a pulsos también presenta ciertos inconvenientes. Existe el peligro de aplicar una dosis de agua menor de la deseada debido a la reducción en la infiltración que suele producirse. En consecuencia, el agricultor ha de controlar la humedad del suelo con mayor frecuencia que si usa métodos tradicionales. Al tratar en muchos casos con automatismos más o menos sofisticados, es necesario emplear un personal más cualificado, siendo además mayor el coste de la instalación. Un manejo poco cuidado podría dar lugar a excesivas pérdidas por escorrentía ya que se reduce la infiltración. Como soluciones a este último problema se proponen técnicas como el recorte de módulo o la interrupción de la entrada de agua una vez ésta haya avanzado sobre un porcentaje suficiente de la superficie a regar, recorte o interrupción que puede controlarse automáticamente.

El fenómeno de la reducción de la velocidad de infiltración no es bien conocido todavía, si bien puede asegurarse que se trata de una combinación de varios mecanismos, entre los que destacan (Blair, 1986): hidratación de partículas de arcilla; consolidación de la capa más superficial del suelo durante el tiempo en el que no se aplica agua; deposición y migración de partículas de suelo, que se traduce en un sellado de la superficie; formación de una capa de aire bajo la superficie del suelo, que quedaría atrapado durante el siguiente período de humectación, reduciendo la conductividad hidráulica del suelo.

\footnotetext{
${ }^{1}$ Universidad de Lleida, Dpto. de Ingeniería Agroforestal. Av/Rovira Roure, 177 -25198-Lleida.e-mail: monserrat@eagrof.udl.

${ }^{2}$ Universidad Pública de Navarra, Dpto. Proyectos e Ingeniería Rural.
}

Artículo publicado en Ingeniería del Agua. Vol.4 Num.2 (junio 1997), páginas 29-36, recibido el 26 de febrero de 1997 y aceptado para su publicación el 22 de mayo de 1997. Pueden ser remitidas discusiones sobre el artículo hasta seis meses después de la publicación del mismo. En el caso de ser aceptadas, las discusiones serán publicadas conjuntamente con la respuesta de los autores en el primer número de la revista que aparezca una vez transcurrido el plazo indicado. 
Los aspectos más importantes que definen las características de un riego a pulsos se tratan a continuación. Atendiendo al criterio del tiempo durante el cual se vierte agua a las unidades de riego, se diferencian fundamentalmente dos métodos de manejo. Uno es el llamado tiempo variable / distancia constante, y el otro el llamado tiempo constante / distancia variable. En el primer caso, se aplica agua durante el tiempo necesario para que el frente húmedo avance en cada pulso una cierta distancia preestablecida. En el segundo, se propone un tiempo constante de aplicación del agua en cada unidad de riego $\mathrm{y}$ en cada pulso, de modo que el agua avanza en cada pulso una distancia diferente hasta alcanzar el punto deseado de la parcela. El número de pulsos es otra de las características propias de un determinado riego. Denominamos tiempo con al tiempo durante el cual se aplica agua a la unidad de riego en un determinado pulso. Por el contrario, tiempo sin es el periodo de tiempo entre pulsos, durante el cual no se aplica agua. Un ciclo está constituido por un tiempo con y un tiempo sin. Tiempo de ciclo es el número de minutos que corresponde a un ciclo. Relación de ciclo es el cociente entre el tiempo con y el tiempo de ciclo.

Son vanados los factores que influyen en el comportamiento del riego a pulsos. Recogemos a continuación algunas conclusiones en este sentido a partir de ensayos realizados en surcos. Respecto a las condiciones edáficas, el riego por pulsos puede no presentar ninguna ventaja, encontrándose que los suelos menos indicados para la aplicación de esta técnica son los de texturas finas y que muestran tendencia al agrietamiento (Goldhamer et al., 1987). En numerosas ocasiones se ha comprobado que en suelos franco arenosos relativamente estables, la velocidad de infiltración mediante la aplicación de pulsos desciende a valores mucho más bajos que en suelos franco arcillosos inestables (Podmore et al., 1982). Es importante señalar que las labores agrícolas pueden modificar de manera decisiva las características del suelo, fundamentalmente por la compactación que implican. Este hecho, así como la presencia de una costra superficial, tiende a reducir la efectividad del riego por pulsos. Por tanto, resultaría que los suelos más indicados para regar a pulsos serían aquellos de textura franca o francoarenosa, de estructura estable, sin costra y que no se agrie-tan. En general, podemos decir que todas aquellas circunstancias edáficas o de manejo del suelo que favorezcan una disminución en la conductividad hidráulica del suelo causan un menor efecto del riego a pulsos, ya que la disminución en la conductividad que esta técnica consigue es en cierto modo producida por dichas circuns-tancias. En este sentido, el comportamiento del riego a pulsos suele ser más positivo durante los primeros rie- gos de la campaña, cuando las condiciones del suelo hacen que las velocidades de infiltración sean relativamente altas (Goldhamer et al., 1987; USDASCS, 1986). Ca-macho (1993) también llegó a conclusiones similares y definió que tiempos con eran más adecuados, así como diversas estrategias de manejo de este tipo de riego.

Coolidge (1981) concluyó que la variación en tiempo con afecta significativamente a los sistemas de riego a pulsos, mientras que la variación en tiempo sin no tiene apenas importancia.

En lo que se refiere a los métodos de manejo del riego a pulsos, de los dos fundamentales anteriormente definidos, parece ser que los mejores resultados se obtienen con el método tiempo variable / distancia constante. Esta afirmación puede resultar especialmente cierta en parcelas de más de 300 m (U.S.D.A-S.C.S., 1986). Un inconveniente a tener en cuenta es que muchos de los equipos de riego por pulsos existentes hoy no están capacitados para utilizar diferentes tiempos de aplicación tal y como exige el método. El método tiempo-cons-tante /distancia variable parece resultar más eficiente sobre surcos de longitud menor de unos 300 m (U.S.D.A-S.C.S., 1986).

La relación de ciclo es una de las variables fundamentales con las que se puede trabajar en riego por pulsos. Los mejores resultados suelen encontrarse con relaciones de ciclo de 1/3, es decir, con un tiempo sin doble duración que el tiempo con (Allen, 1980; Poole, 1981). Generalmente, las grandes dosis de agua se aplican más eficientemente con largos tiempos de ciclo, y las pequeñas, con cortos. Tiempos de ciclo demasiado cortos pueden causar que no se infiltre toda el agua aportada antes de la aplicación del nuevo gasto correspondiente al pulso siguiente, con lo que obtenemos resultados equivalentes a un riego continuo. Con tiempos de ciclo demasiado cortos se corre también el riesgo de que el frente de avance no pueda alcanzar el final.

El riego superficial por tablares, en sus diferentes modalidades, es ampliamente utilizado en diferentes zonas, resultando imprescindible para cultivos que no se adaptan a plantación-sembrado en hilera. Las peculiares características del riego por tablares, como el elevado volumen almacenado superficialmente, hacen imposible extrapolar los resultados del riego por pulsos en surcos al riego por pulsos en tablares. Se hace por tanto necesario proceder a experimentaciones específicas. Como ya se ha indicado, existe muy poca información a cerca del riego a pulsos en tablares. De todos modos, Ismail et al. (1985) (citado en Westesen y Biglen, 1986) encontraron un incremento de la eficiencia de la aplicación usando riego a pulsos frente a riego continuo. Los tablares eran de $3 \mathrm{~m} \times 102 \mathrm{~m}$, con una pendiente del 1,5\%. La eficiencia de la aplicación pasó del $67 \%$ con riego continuo al $82 \%$ con riego a pulsos, y el agua alcanzó el final del tablar en $35 \mathrm{~min}$ con riego a pulsos, mientras que con riego continuo invirtió $56 \mathrm{~min}$. Westesen y Biglen (1986) encontraron resultados positivos en ensayos con tablares de 30,5 m x $762 \mathrm{~m}$, sobre suelo franco arcilloso, con una pendiente media del $0,31 \%$. Para un primer riego, seleccionaron un tiempo con de $100 \mathrm{~min}$ y un tiempo sin de $60 \mathrm{~min}$, siendo el caudal de entrada de $1471 / \mathrm{s}$. Se necesitaron $6.6 \mathrm{~h}$ de aplicación de agua para completar el riego con el caudal continuo, mientras que el tiempo de aplicación (tiempo con) necesario en el riego a pulsos fue de 5 horas (tres pulsos de $100 \mathrm{~min}$ ). Durante un segundo riego posterior, con el mismo 
caudal, se aplicaron también tres pulsos, los dos primeros con tiempos con y sin iguales y de $90 \mathrm{~min}$, y el tercero con tiempos con y sin también iguales pero de $60 \mathrm{~min}$. En esta ocasión, fueron necesarias $7 \mathrm{~h}$ de aplicación continua, mientras que bastaron $4 \mathrm{~h}(90+90+60 \mathrm{~min}) \mathrm{de}$ aplicación en el riego a pulsos para completar el avance.

El objetivo del presente trabajo es comprobar, bajo variadas condiciones de suelo y manejo, la aplicabilidad de la técnica de riego a pulsos en riego superficial por tablares (canteros) con pendiente

\section{MATERIAL Y MÉTODOS}

Se han realizado ensayos en cuatro parcelas situadas en los términos municipales de Penelles (P), Castell del Remei (CR), Arbeca (A), y Lleida (Ll), en adelante se abreviarán con la letra entre paréntesis, todos ellos de la provincia de Lleida. Las parcelas P. CR. y A corresponden a explotaciones comerciales de la zona regable de los canales de Urgell, mientras que la Ll estaba situada en una finca experimental.

En la Tabla 1 se muestran las características principales de las parcelas y de los riegos realizados, pudiéndose comprobar las variadas condiciones de los ensayos.

Una característica a destacar del suelo de la parcela $\mathbf{L l}$. es su tendencia al encostramiento y posterior agrietamiento después de un riego sobre terreno labrado.

En las parcelas comerciales (P, CR, y A) la condición de contorno aguas abajo era sin escorrentía libre, mientras que en Ll ésta se permitió.

En cada parcela se escogieron tres tablares, dos para la prueba de pulsos entre los que se iba alternando el agua, y otro para la prueba de continuo. Cada prueba consistía en regar en un mismo día, y bajo las mismas condiciones, el tablar de continuo y los dos de pulsos. Como puede apreciarse en la Tabla 7, en la parcela CR se evaluaron cuatro riegos, en $\mathbf{L l}$ dos, y en $\mathbf{P}$, y $\mathbf{A}$ se evaluó un riego.

El método de manejo que se intentó aplicar en las prue- bas de pulsos fue el de distancia constante / tiempo variable ya que de esta manera resulta más sencillo prefijar el número de pulsos a realizar en una prueba. Sin embargo, al realizar el riego en las parcelas P, CR, y A, ocurrió que el agua, después del corte de un determinado pulso una vez alcanzada la posición predeterminada, avanzaba una distancia considerable, debido a la importante cantidad de agua almacenada superficialmente, resultando que realmente el manejo aplicado fue de distancia variable / tiempo variable. En los riegos de las parcelas P, CR, y A, el tiempo de corte del agua (fin de la aplicación en el riego) se realizó cuando el agua avanzaba tres cuartas partes de la longitud total del tablar, tanto en riego a pulsos como en continuo. En la parcela Ll, el avance continuaba pocos metros una vez cortado el aporte, por lo que en este caso sí se aplicó realmente el método distancia constante / tiempo variable. El tiempo de corte en esta parcela se estableció en el momento en que el agua alcanzaba el extremo final de la misma.

La relación de ciclo no era prefijada "a priori" ya que el criterio para empezar un nuevo ciclo era cuando se había producido el receso en el ciclo anterior.

En cada riego se medía el caudal de entrada y el caudal de escorrentía (si existía) mediante aforadores portátiles de cresta ancha. Los tiempos de avance y receso se controlaban a intervalos de $10 \mathrm{~m}$. Por último, y con el fin de obtener una estimación de la aspereza o rugosidad, se procedió a medir el calado del agua en la cabecera de los tablares al final del riego, lo que permite calcular el coeficiente $n$ de Manning, suponiendo un régimen permanente para el flujo del agua.

Para evaluar las posibles ventajas o inconvenientes de la técnica de riego a pulsos respecto a la de continuo se han escogido para su comparación algunas variables del riego, a las que se denomina indicadores de un riego. Dichos indicadores se pueden dividir en dos tipos: directos e indirectos. Indicadores directos son aquellos que se pueden medir directamente en campo, como tiempo con o tiempo de aplicación necesario para llegar al final de la parcela y lámina media infiltrada. Indicadores in-

\begin{tabular}{|c|ccccccc|}
\hline PRUEBA & $\begin{array}{c}\text { Longitud } \\
(\mathbf{m})\end{array}$ & $\begin{array}{c}\text { Anchura } \\
(\mathbf{m})\end{array}$ & $\begin{array}{c}\text { Caudal } \\
(\mathbf{I} / \mathbf{s} / \mathbf{m})\end{array}$ & $\begin{array}{c}\text { Pendiente } \\
(\mathbf{\%})\end{array}$ & $\begin{array}{c}\text { Textura } \\
(\mathbf{U S D A})\end{array}$ & $\begin{array}{c}\mathbf{N}^{\circ} \text { Riego } \\
(\text { () }\end{array}$ & Cultivo \\
\cline { 2 - 7 } P & 228 & 26.8 & 3.44 & 0.1 & F-Ar & $1^{\circ}$ & Trigo \\
CR-1 & 370 & 24.8 & - & 0.09 & F-Arc-L & $1^{\circ}$ & Alfalfa \\
CR-2 & 370 & 24.8 & 5.69 & 0.09 & F-Arc-L & $2^{\circ}$ & Alfalfa \\
CR-3 & 370 & 24.8 & 6.54 & 0.09 & F-Arc-L & $5^{\circ}$ & Alfalfa \\
CR-4 & 370 & 24.8 & 5.33 & 0.09 & F-Arc-L & $6^{\circ}$ & Alfalfa \\
A & 223 & 7.2 & 14.66 & 0.1 & F-Ar & $4^{\circ}$ & Melocot. \\
LI-2 & 190 & 5.26 & 1.54 & 0.24 & F & $1^{\circ}$ & s/cultivo \\
LI-3 & 190 & 4.97 & 1.50 & 0.24 & F & $2^{\circ}$ & s/cultivo \\
\hline
\end{tabular}

(*) Orden cronológico que ocupó el riego analizado con respecto a los otros realizados ese año en la misma parcela

Tabla 1: Características principales de las parcelas y el caudal de los ensayos realizados 
directos son aquellos que para ser obtenidos precisan del conocimiento de la función de infiltración. Por ejemplo, el coeficiente de uniformidad de Christiansen, y el perfil del agua infiltrada. La distinción anterior se debe a que si en alguna prueba no es posible obtener la función de infiltración, se puede realizar una comparación válida del riego a pulsos respecto al continuo. No obstante el conocimiento de la función de infiltración permite estudiar con más profundidad lo que sucede durante el riego.

La función de infiltración se ha caracterizado mediante la ecuación de Kostiakov simple, $\mathrm{z}=\mathrm{k} \mathrm{t}_{\mathrm{c}}{ }^{\mathrm{a}}$, donde $\mathrm{z}$ es altura de agua infiltrada acumulada, $\mathrm{k}$ y a parámetros empíricos de ajuste y $t_{c}$ tiempo de oportunidad o de contacto. En el caso de riego a pulsos corresponde una función para cada tramo con las mismas condiciones de humectación (suelo seco, mojado una vez,.etc....). El tiempo de contacto se considera desde el inicio del pulso y no desde el inicio del riego, esto hace que las funciones de infiltración no sean directamente comparables, ya que parten de un origen de tiempos diferente, no obstante a efectos de estimación de la lámina infiltrada esto no afecta . Los parámetros de dicha ecuación se han obtenido mediante el programa informático OPTYM0 v.5 (Monserrat, 1994), tanto para el riego continuo (suelo seco) como para el riego a pulsos (suelo mojado una vez). En dicho programa se utiliza un método inverso basado en el modelo hidrológico o de balance de volumen del riego por tablares que mediante un proceso iterativo encuentra los parámetros de infiltración que mejor verifican la ecuación de continuidad planteada en varios instantes durante el avance. El programa presenta la particularidad respecto a otros métodos basados en el modelo hidrológico en que hace una estimación del volumen superficial a partir de datos obtenidos mediante el modelo de inercia nula. Los datos de entrada son :

- caudal

- pendiente

- aspereza o rugosidad hidráulica

- tiempos de avance.

\section{RESULTADOS Y DISCUSIÓN}

En la Tabla 2 se exponen los principales resultados de los ensayos de comparación:

En cuanto a los indicadores directos (tiempo con y lámina media infiltrada) es de destacar que en todas las pruebas, excepto en la Ll-2, el riego continuo ha mejorado el de pulsos, a pesar de que se dan algunas de las condiciones teóricamente favorables, como el hecho de que las texturas de los suelos de las parcelas $\mathbf{P}, \mathbf{C R}$, y A responden a las citadas en la bibliografía como más deseables. En efecto, en la prueba $\mathbf{L l - 2}$ el tiempo con necesario ha pasado de 249 min para el riego continuo a 189 para el riego a pulsos, y la lámina media infiltrada de 0.13 a $0.10 \mathrm{~m}$ respectivamente. Así, las condiciones en que la técnica de pulsos ha mejorado el riego son: suelo franco recién labrado, baja rugosidad hidráulica, y bajo caudal unitario. En las mismas condiciones, en dicha parcela en el riego siguiente no se obtuvieron mejoras, hecho que puede explicarse por el asentamiento del suelo que tiene lugar después de un riego, lo que disminuye su velocidad de infiltración. De este modo, el efecto de reducción de velocidad de infiltración que induce el riego por pulsos ya lo ha producido el propio asentamiento. En este suelo, como ya se ha indicado, hay además una clara tendencia al encostramiento y agrietamiento que se puso de manifiesto después del primer riego. En el resto de las pruebas el riego a pulsos ha mostrado un comportamiento peor que el continuo, incluso en el primer riego del año.

Para explicar la causa de los resultados obtenidos vamos a analizar la evolución de alguno indicadores indirectos durante el riego, en concreto, la evolución del volumen infiltrado total respecto a la distancia avanzada. Para ello necesitaremos conocer las funciones de infiltración para riego continuo y riego a pulsos. A modo de ejemplo estudiaremos primeramente en detalle la prueba CR-2.

En las Figuras 1,2 y 3 se han representado respectivamente las curvas de avance y receso, del riego continuo, a pulsos, así como la comparación de las curvas de avance de ambos, para dicha prueba.

\begin{tabular}{|c|c|c|c|c|c|c|c|c|c|c|c|}
\hline \multirow{3}{*}{$\begin{array}{c}\text { PRUEBA } \\
P\end{array}$} & \multirow{3}{*}{\begin{tabular}{|c|}
$\mathbf{N}^{\mathbf{0}}$ Pulsos \\
3 \\
\end{tabular}} & \multicolumn{4}{|c|}{$\begin{array}{l}\text { Distancia de corte } \\
\text { (m) }\end{array}$} & \multirow{2}{*}{$\begin{array}{c}\begin{array}{c}\text { Coef. } \\
\text { Manning }\end{array} \\
n\end{array}$} & \multicolumn{2}{|c|}{$\begin{array}{l}\text { Tiempo"con" } \\
\text { (min.) }\end{array}$} & \multirow{2}{*}{\begin{tabular}{|c|}
$\begin{array}{c}\text { Alt. med } \\
\text { infiltrada (m) }\end{array}$ \\
Cont Pulsos \\
\end{tabular}} & \multicolumn{2}{|c|}{$\begin{array}{c}\text { Coef. } \\
\text { Uniformidad } \\
\text { (CU) }\end{array}$} \\
\hline & & Pulso & Pulso & Pulso & 3 Cont. & & Cont. & Pulsos & & Cont. & Pulsos \\
\hline & & 30 & 75 & 170 & 170 & 0.234 & 129 & 146 & 0.1170 .129 & 97 & 75.5 \\
\hline CR-1 & 3 & 60 & 110 & 260 & 260 & - & 101 & 141 & - & - & - \\
\hline CR-2 & 2 & 100 & 260 & - & 260 & 0.204 & 120 & 138 & 0.1110 .122 & 97.3 & 89.4 \\
\hline CR-3 & 2 & 120 & 240 & - & 240 & 0.188 & 90 & 116 & 0.0960 .115 & 93.8 & 85.8 \\
\hline CR-4 & 2 & 80 & 240 & - & 240 & 0.221 & 106 & 131 & 0.0920 .106 & 96.7 & 89.9 \\
\hline $\mathbf{A}$ & 9 & 50 & 170 & - & 170 & 0.056 & 34 & 41 & 0.1340 .157 & 97 & 75.5 \\
\hline LI-2 & 3 & 60 & 120 & 190 & 190 & 0.02 & 249 & 189 & 0.1300 .100 & 88.1 & 85.9 \\
\hline LI-3 & 3 & 60 & 120 & 190 & 190 & 0.02 & 168 & 182 & 0.0790 .088 & 97.8 & 95.9 \\
\hline
\end{tabular}

Los recuadros sombreados señalan la técnica que ha obtenido el valor mejor de un indicador para una determinada prueba comparativa. 


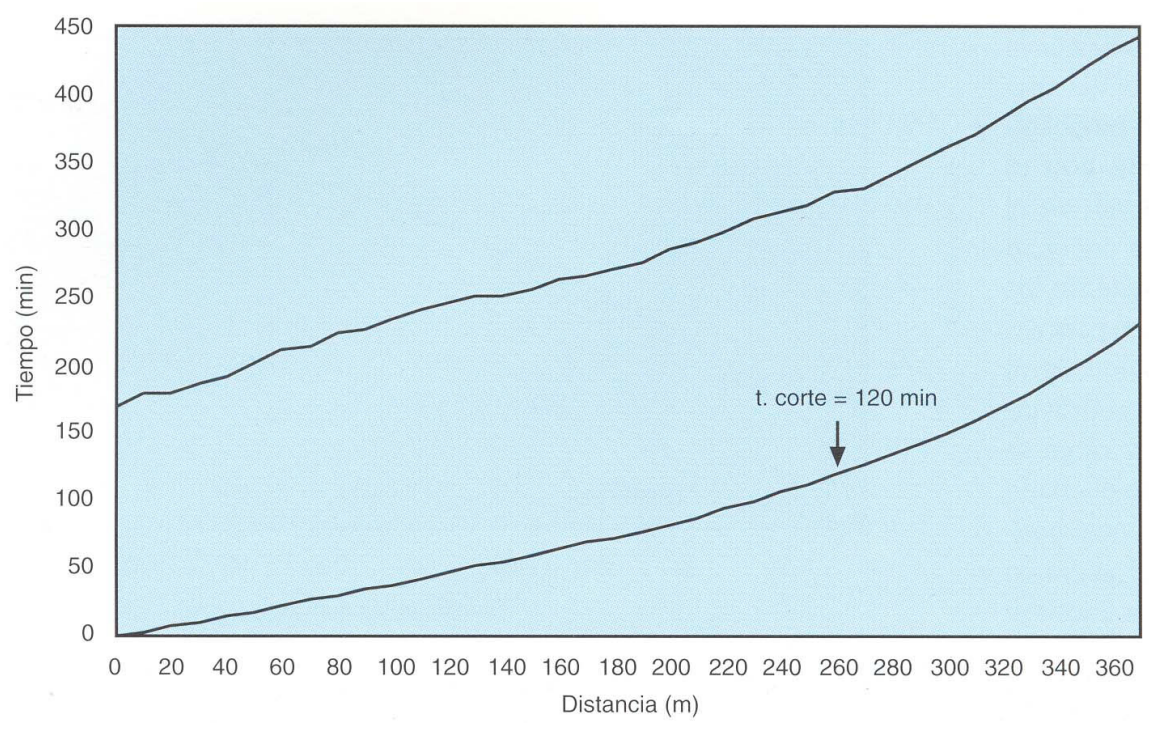

Figura 1: Curvas de avance y receso para la prueba de continuo CR-2

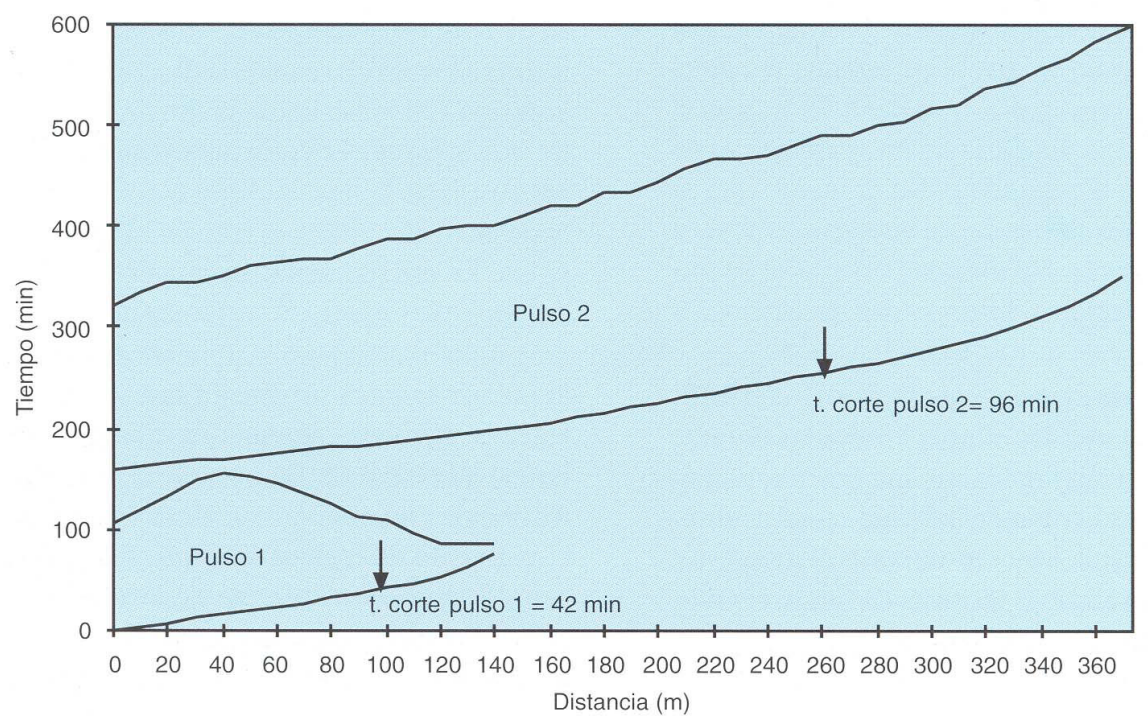

Figura 2: Curvas de avance y receso para la prueba de pulsos $C R-2$

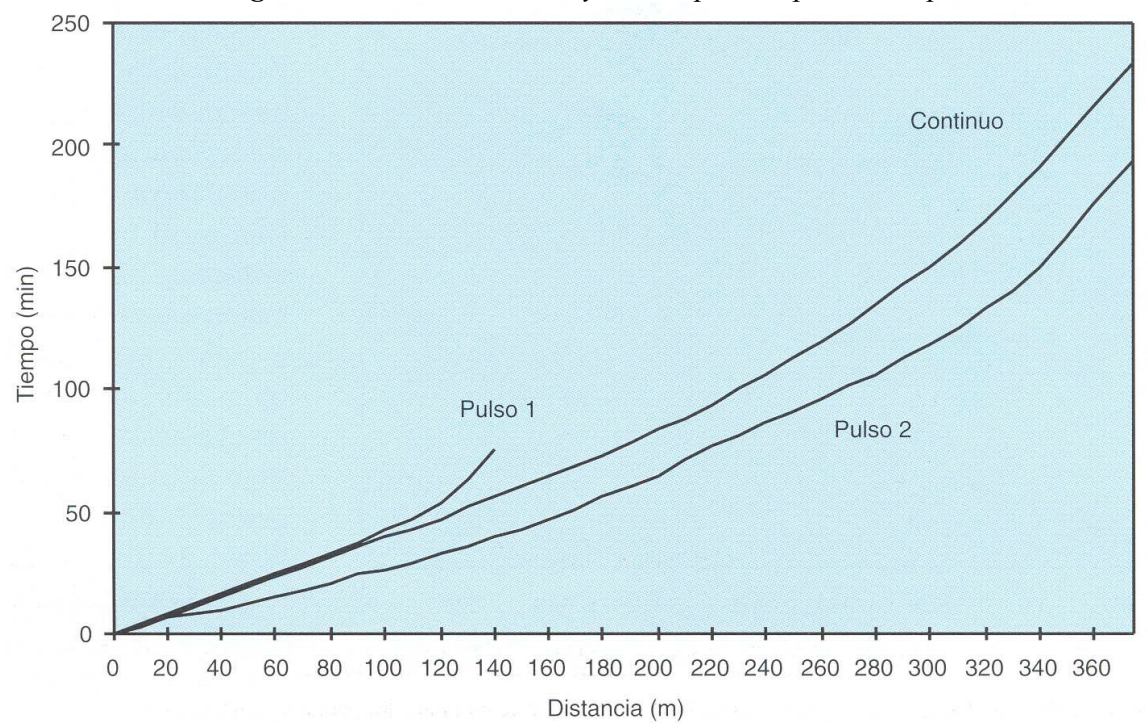

Figura 3: Comparación de las curvas de avance entre el riego continuo y pulsos 
En la Figura 4 se presenta la función de infiltración del suelo seco y la del suelo humectado una vez obtenida con el programa OPTYMO v.5, en el rango de tiempos de contacto utilizados para el ajuste de los parámetros. La función para caracterizar la infiltración en el pulso 2, para el suelo ya previa-mente mojado de este riego se ha obtenido aplicando el citado programa a los datos correspondientes al avance del segundo pulso de la prueba en cuestión sobre la zona ya mojada del pulso 1. Para comprobar la fiabilidad de las funciones de infiltración obtenidas se ha calculado el error en la estimación del volumen infiltrado con la función de infiltración respecto al volumen entrado ya que la escorrentía era nula. El resultado obtenido en el pulso 1 era del $5.58 \%$, y en el segundo pulso del $7.65 \%$. En dicha figura se aprecia cómo la lámina infiltrada en el segundo pulso es menor que en el primero,

para un mismo tiempo de contacto contado a partir del inicio del pulso. No obstante esto no se ha traducido en una disminución del tiempo con necesario, aspecto que trataremos de explicar a continuación.

A partir de los datos de avance, y de la función de infiltración se pueden calcular las alturas infiltradas durante el avance. Posteriormente, mediante integración numé-rica, puede calcularse el volumen infiltrado total acumu-lado en un determinado instante durante el riego. La Figura 5 muestra, para ambas técnicas de riego, el volu-men por unidad de anchura de tablar que se ha infiltrado en función de las posiciones que va alcanzando el frente de avance. Vemos que durante el avance del primer pulso $(\mathrm{x}<100 \mathrm{~m})$

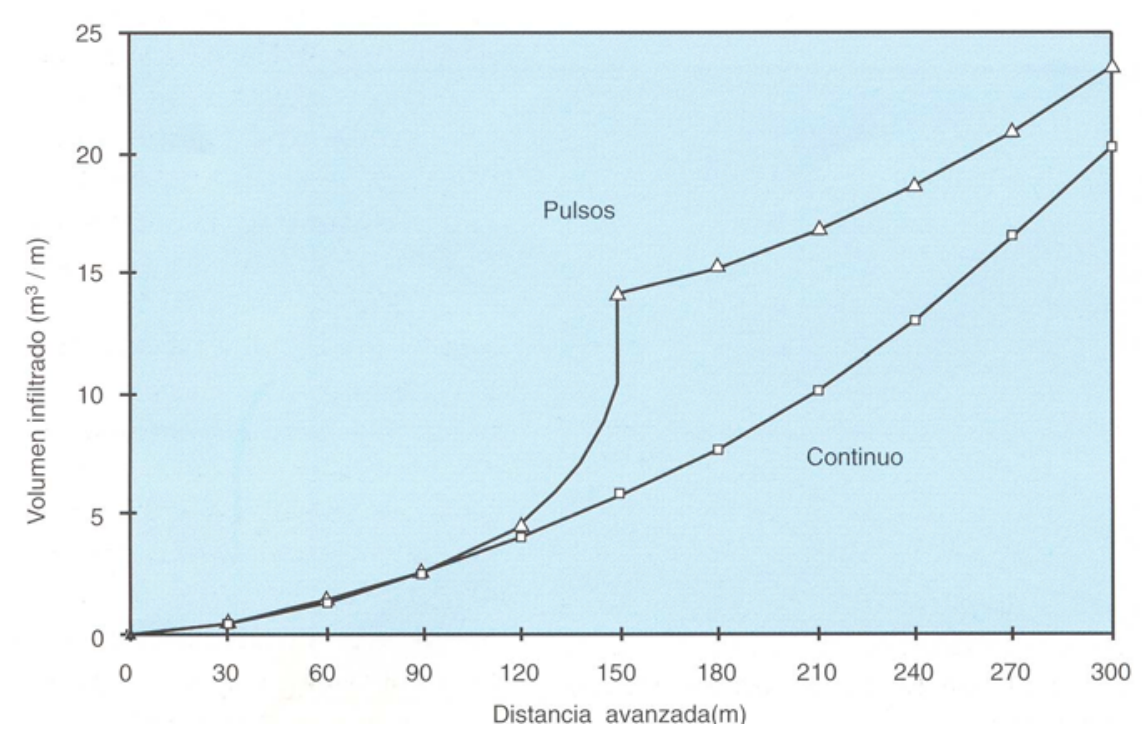

Figura 5: Evolución del volumen infiltrado total en función e la distancia avanzada para el ensayo CR-2

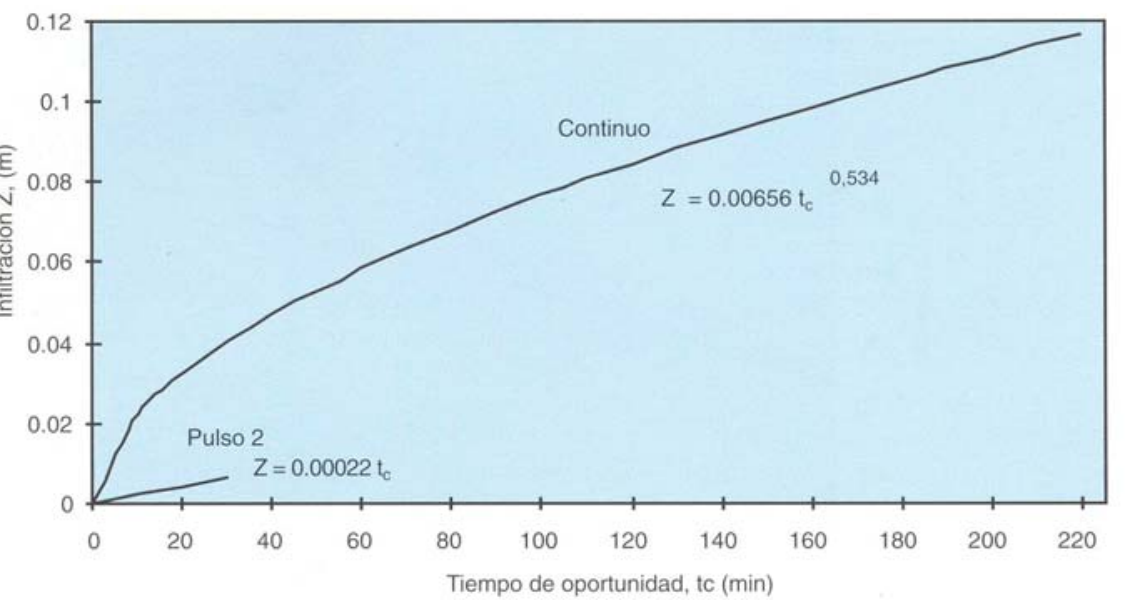

Figura 4.- Función de infiltración para suelo seco (Continuo) suelo mojado una vez (Pulso 2) representadas enel rango de tiempos de contado utilizado para el ajuste de las mismas

coinciden las dos líneas, y por tanto se ha infiltrado la misma cantidad de agua. Para distancias mayores de $100 \mathrm{~m}$ las líneas comienzan a diverger: se infiltra mas agua en el riego a pulsos que en el riego continuo para avanzar la misma distancia. La causa hay que buscarla en lo que ocurre al realizar el corte al fin del primer pulso. En ese momento se encuentra sobre la superficie del suelo un importante volumen de agua. Por definición del propio método, no se aplicará el siguiente pulso hasta que no se haya producido una recesión completa del anterior. Por tanto, durante ese tiempo, se infiltra un volumen adicional de agua en el riego a pulsos que se corresponde con el volumen almacenado en superficie en el momento de realizar el corte. Además, toda ese agua se infiltra sin que el frente de avance adelante su posición de manera importante, ya que el aporte de agua se ha interrumpido. En el avance del segundo pulso se infiltra una pequeña cantidad en el tramo previamente humectado $\left(0.728 \mathrm{~m}^{3}\right.$ para $\left.\mathrm{x}<140 \mathrm{~m}\right)$. A partir de entonces el volumen de agua infiltrado por unidad de distancia avanzada es menor en pulsos que en continuo, consecuencia de que ha habido una disminución de la tasa de infiltración en el tramo humectado y el avance es más rápido. A pesar de haberse producido una disminución de la tasa de infiltración, esta no ha sido suficiente para compensar el efecto negativo del volumen superficial infiltrado en el primer pulso. El presente análisis se detiene a $10300 \mathrm{~m}$ ya que es durante el avance donde mejor se manifiesta el efecto de los pulsos. A partir de esta distancia empieza el receso en el origen (Figura 2). 
La figura 6 ilustra el mismo análisis para la prueba $\mathrm{P}$, en la que el primer pulso fue más corto. Se aprecia cómo llegan a igualarse los volúmenes infiltrados para una determinada distancia durante el segundo pulso, pero la prueba de pulsos no llega a conseguir menores volúmenes infiltrados.

La discusión realizada con las pruebas CR-2 y $\mathbf{P}$ es extensible a las otras pruebas, excepto a la Ll2. El análisis de los párrafos anteriores puede ayudar también a explicar el éxito de la prueba Ll-2. En efecto, en ella el caudal unitario era muy bajo en relación a las realizadas en las otras parcelas, y también era bajo el valordel coeficiente $\mathrm{n}$ de Manning, factores ambos que contribuyen a un bajo volumen superficial.

En la parcela CR se ensayaron diversas alternativas de manejo. En el primer riego se aplicaron tres pulsos, pero dado el mal resultado obtenido, se probó con dos pulsos y diferentes longitudes avanzadas para el primer pulso. Los resultados comparativamente al riego continuo mejoraron un poco pero sin superarlo. La estrategia de riego utilizada en las pruebasP, CR, y A de cortar el agua a la misma distancia en el riego a pulsos que en el continuo se podía haber mejorado cortando el agua antes en los pulsos, debido a que su «potencial» de avance es mayor que para esa misma distancia en riego continuo, pues el suelo humectado varias veces infiltra menos y por tanto el volumen superficial es mayor.

Otro aspecto a analizar es la comparación del perfil de agua infiltrada al final del riego obtenido con ambas

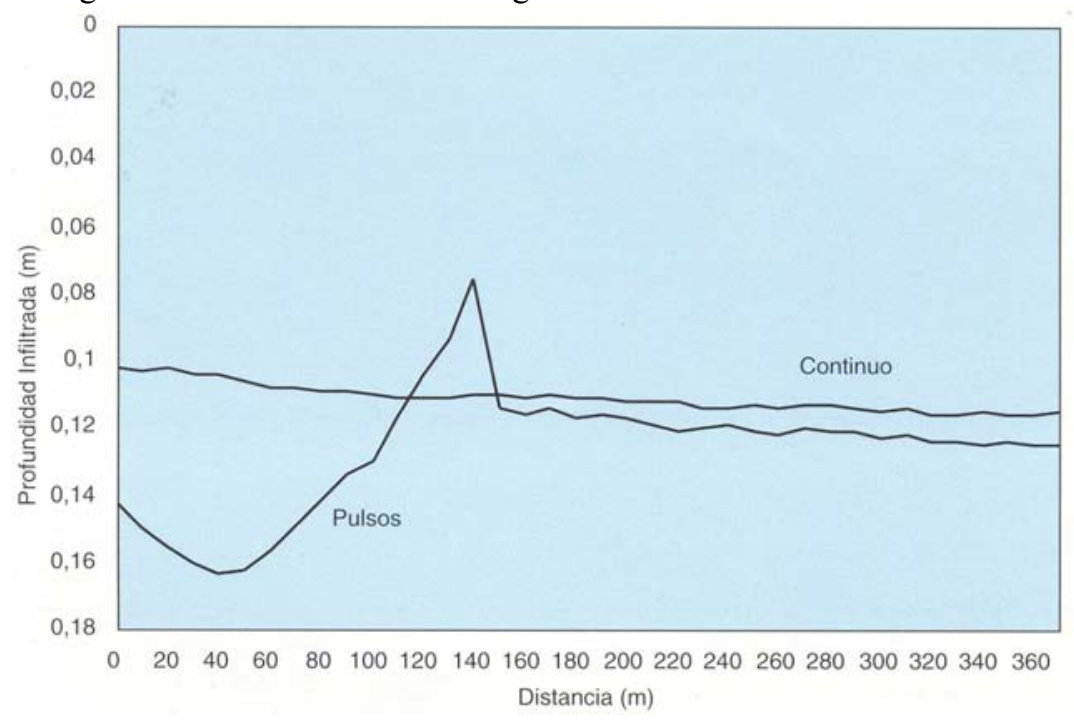

Figura 7: Perfiles de agua infiltrada para el riego continuo y el riego a pulsos en el ensayo CR-2
Figura 6: Evolución del volumen infiltrado total en función de la distancia avanzada para el ensayo $P$

técnicas. En la Figura 7 se aprecia claramente la menor uniformidad del riego a pulsos, debida al fenómeno de infiltración del volumen superficial. En consecuencia, los valores del coeficiente de uniformidad (Tabla 2) resultan mayores en las pruebas de riego continuo respecto a la de pulsos.

\section{CONCLUSIONES}

En la mayoría de los ensayos realizados el riego a pulsos no mejora los resultados del riego continuo en condiciones de riego por tablares. Se ha observado un efecto reductor de la infiltración en las pruebas de riego a pulsos en tablares. No obstante, esta reducción sólo se traduce en una disminución del tiempo de avance cuando hay un volumen superficial bajo, que parece ser el fac-tor controlador de la efectividad del riego a pulsos en tablares. En las pruebas realizadas estas circunstancias. se dieron para caudales unitarios bajos $(1.51 / \mathrm{s} / \mathrm{m})$, rugosidad hidráulica baja $(\mathrm{n}=0.02)$, y suelo recién labrado. La aplicabilidad de esta técnica en riego por tablares se restringe a condiciones similares a las anteriormente descritas. El efecto de la textura y número de pulsos no fue determinante para la mejora de los resultados respecto al riego continuo.La técnica de manejo de distancia constante/tiempo variable es adecuada cuando se desconoce el comportamiento de la parcela frente a los pulsos. En riego por tablares, no obstante, es difícil prefijar la distancia avanzada debido al avance considerable que ocurre después de cortar el agua. 


\section{REFERENCIAS}

Allen, N. L. (1980). Advance Rates in Forrow Irrigation for Cycled Flow. Ph.D. diss. Utah State University, Logan.

Blair, A.W. ( 1986) Surge flow irrigation infiltration. In Surge Flow Irrigation Field Guide. U.S.D.A.S.C.S.

Coolidge, P. S. (1981) Advance Rates Under Automated Pulsed Flow Irrigation System. Ph.D. diss. Utah State University, Logan.

Camacho, E. (1993) Caracterización, optimización y manejo del riego por surcos mediante pulsaciones intermitentes. Tesis Doctoral. Universidad de Córdoba.

Goldhamer, D.A., H.A. Mohammad and R.C. Phene (1987) Surge vs. continous-flow irrigation. $\mathrm{Ca}-$ lifornia Agriculture, Sep-Oct: 29-32.

Izuno, F.T., T.H. Podmore and H.R. Duke, (1985) Infiltration under surge irrigation. Transactions of ASAE 28 (2): 517-521.
Monserrat, J., (1994). Solución al problema inverso del riego por tablares mediante un modelo hidrológico mixto. Tesis Doctoral. E.T.S.I.A., Universidad de Lleida.

Podmore, T. H. and H. R. Duke, (1982). Field evaluation of Surge Flow Irrigation. Paper 82-2012. A.S.A.E, Madison, Wisconsin.

Poole, G. J. (1981). Infiltration and Advance Under Surge Flow Irrigation. Ph.D. diss. Utah State University, Logan.

Stringham, G. E.and J. Keller, (1979). Surge Flow Automatic Irrigation. Irrigation and Drainage Divi-sion Speciality Conference, A. S. C. E., Albuquerque, New Mexico.

U.S.D.A - S.C.S. (1986). Surge Flow Irrigation Field Guide. $32 \mathrm{pp}$.

Walker, W.R and G. V. Skogerboe, (1987). Surface irrigation: Theory and Practice. Prentice-Hall, INC, Englewood Clifs, New Yersey. 386 pp

Westesen, G. L and D. K. Biglen, (1986). Surge flow border irrigation trials. A.S.A.E. paper $\mathrm{n}^{\circ}$ : 862082. 